\title{
Enzymatic N-Acylation Step Enables the Biocatalytic Synthesis of Unnatural Sialosides
}

\author{
Pedro Laborda+, Yong-Mei Lyu+, Fabio Parmeggiani, Ai-Min Lu, Wen-Jiao Wang, Ying-Ying Huang, \\ Kun Huang, Juan Guo, Li Liu*, Sabine L. Flitsch*, and Josef Voglmeir*
}

\begin{abstract}
Selective $\mathrm{N}$-acylation: Chitin is one of the most abundant and cheaply available biopolymers in Nature. Chitin has become a valuable starting material for many biotechnological products through manipulation of its $\mathrm{N}$-acetyl functionality, which can be cleaved under mild conditions using the enzyme family of de- $N$-acetylases. However, the chemo-selective enzymatic re-acylation of glucosamine derivatives, which can introduce new stable functionalities into chitin derivatives, is much less explored. Here we describe an acylase (CmCDA from Cyclobacterium marinum) that catalyzes the $\mathrm{N}$ acylation of glycosamine with a range of carboxylic acids under physiological reaction conditions. This biocatalyst closes an important gap in allowing for the conversion of chitin to complex glycosides, such as C5-modified sialosides, through the use of highly selective enzyme cascades.

Carbohydrate active enzymes (CAZy) have become valuable catalysts for the highly selective production of complex natural and unnatural glycans and their use in one-pot enzyme cascades is attractive [1]. In particular, glucosamine derivatives are fundamental building blocks for the synthesis of a range of hexosamines and sialylated glycoconjugates [2] and as bioorthogonal chemical reporters for metabolic glycan labelling and engineering [3] as illustrated in Scheme 1. Glucosamine (2) itself is a valuable food additive which can be generated from chitin through chemical or enzymatic depolymerization to 1 and de-acetylation [4]. Subsequent epimerases (to generate the galactose and mannose derivatives 4 and 5 respectively) and further elaboration to sialic acids (6) and glycoconjugates can also be achieved enzymatically. However, there
\end{abstract}

[*] Dr. Pedro Laborda, Yong-Mei Lyu, Ying-Ying Huang, Wen-Jiao Wang, Juan Guo, Prof. Li Liu, Prof. Josef Voglmeir

Glycomics and Glycan Bioengineering Research Center (GGBRC)

College of Food Science and Technology

Nanjing Agricultural University, 1 Weigang, 210095 Nanjing (China)

Email: lichen.liu@njau.edu.cn josef.voglmeir@njau.edu.cn

Web: www.ggbrc.com

Prof. Pedro Laborda

School of Life Sciences

Nantong University, 19 Qixiu Road, 226019 Nantong (China)

Dr. Ai-Min Lu

College of Sciences

Nanjing Agricultural University, 1 Weigang, 210095 Nanjing (China)

Dr. Fabio Parmeggiani, Dr. Kun Huang, Prof. Sabine L. Flitsch

School of Chemistry \& Manchester Institute of Biotechnology

The University of Manchester

131 Princess Street, M1 7DN, Manchester (UK)

Email: sabine.flitsch@manchester.ac.uk

Web: www.flitschlab.com

$\left.{ }^{+}\right]$These authors contributed equally to this work.

Supporting information for this article is given via a link at the end of the document. remains a gap in the enzyme toolkit in that the acylation of glucosamine 2 needs to be performed chemically, requiring activation of carboxylic acids or stoichiometric coupling agents and protecting strategies [5].

Hence, the enzymatic synthesis of $\mathrm{N}$-acylated glucosamine derivatives directly from glucosamine and unprotected carboxylic acids would allow for the generation of target structures 3-6 and their derivatives using enzyme cascades directly from chitin (Scheme 1). In biosynthesis, $\mathrm{N}$-acetylation is often mediated through the generation of acetyl precursors such as acetyl coenzyme A or acetyl phosphate, or acetyl adenine [6] and cannot directly utilize glucosamine 2, but require activated glucosamine-6-phosphate as the acceptor substrate [7]. A more attractive strategy is the reversible enzymatic amine acyl exchange of amines and free carboxylic acids (Scheme 1, compound $\mathbf{2}$ to $\mathbf{3}$ using free acid as the acylating agent), thus directly replacing the chemical step. Such enzymatic reaction has been previously described for protease-catalyzed $\mathrm{N}$-acylation reactions of peptides or urea [8], but to our knowledge, there is no example of the $\mathrm{N}$-acylation of glucosamine. An enzyme-catalyzed approach would not require activation of the carboxylate and in addition has the potential to be highly selective for glucosamine in the presence of other biogenic amines.

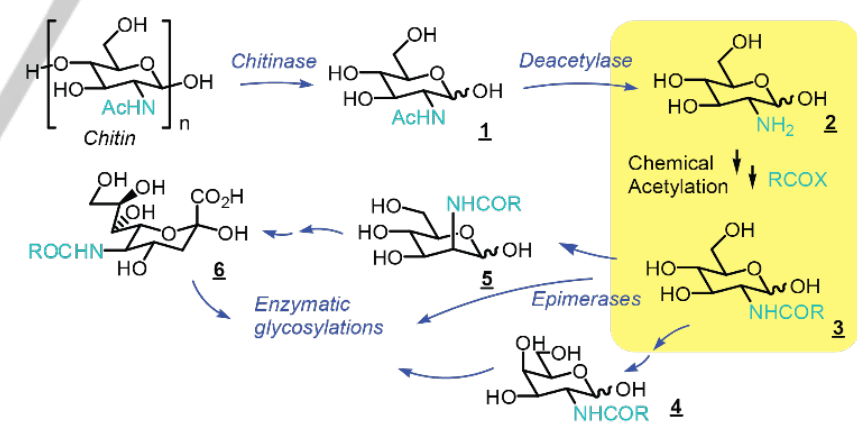

Scheme 1. Current enzyme cascades for the conversion of chitin from biomass to complex glycoconjugates, which still require chemical acylation strategies for amino sugars. Here we address the challenge of achieving selective acylation by enzyme catalysis.

In the absence of any obvious candidate enzymes from the literature, we looked for promiscuous activity of known biocatalysts. We previously described a recombinant chitin de- $\mathrm{N}$-acetylase $\mathrm{CmCDA}$ that is able to specifically and efficiently deacylate $\mathrm{N}$-acylglucosamine derivatives (including GICNAc), whereas no activity towards other carbohydrates such as chitobiose, ManNAc or GalNAc was detected [4].

We also noted similarities in active site residues between CmCDA and a previously reported peptidoglycan deacetylase [9]. The relevant catalytic amino acids appear to be conserved between 


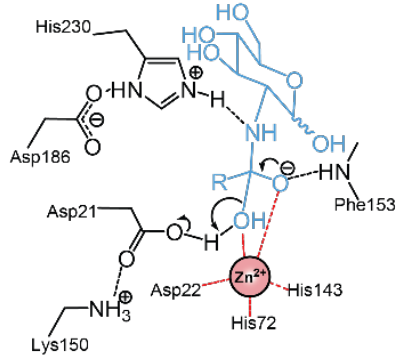

Scheme 2. Proposed $\mathrm{N}$-acylation mechanism of glucosamine by CmCDA. The tetrahedral oxyanion intermediate is shown in the catalytic site of CmCDA. both enzymes, including the His-His-Asp zinc-binding triad coordinating the $N$-acetyl group, as well as the respective histidine and aspartic acid residues acting as the catalytic acid and base. The active site residues suggest acylation via the described tetrahedral oxyanion intermediate which would also allow for the reverse the reaction, i.e. the $N$-acylation reaction of glucosamine (1) with a carboxylic acid (Scheme 2).

Table 1. CmCDA catalysed $\mathrm{N}$-acylation of $\mathbf{1}$ and transacylation of $\mathbf{2}$ from carboxylic acids $8-13 .^{[a]}$

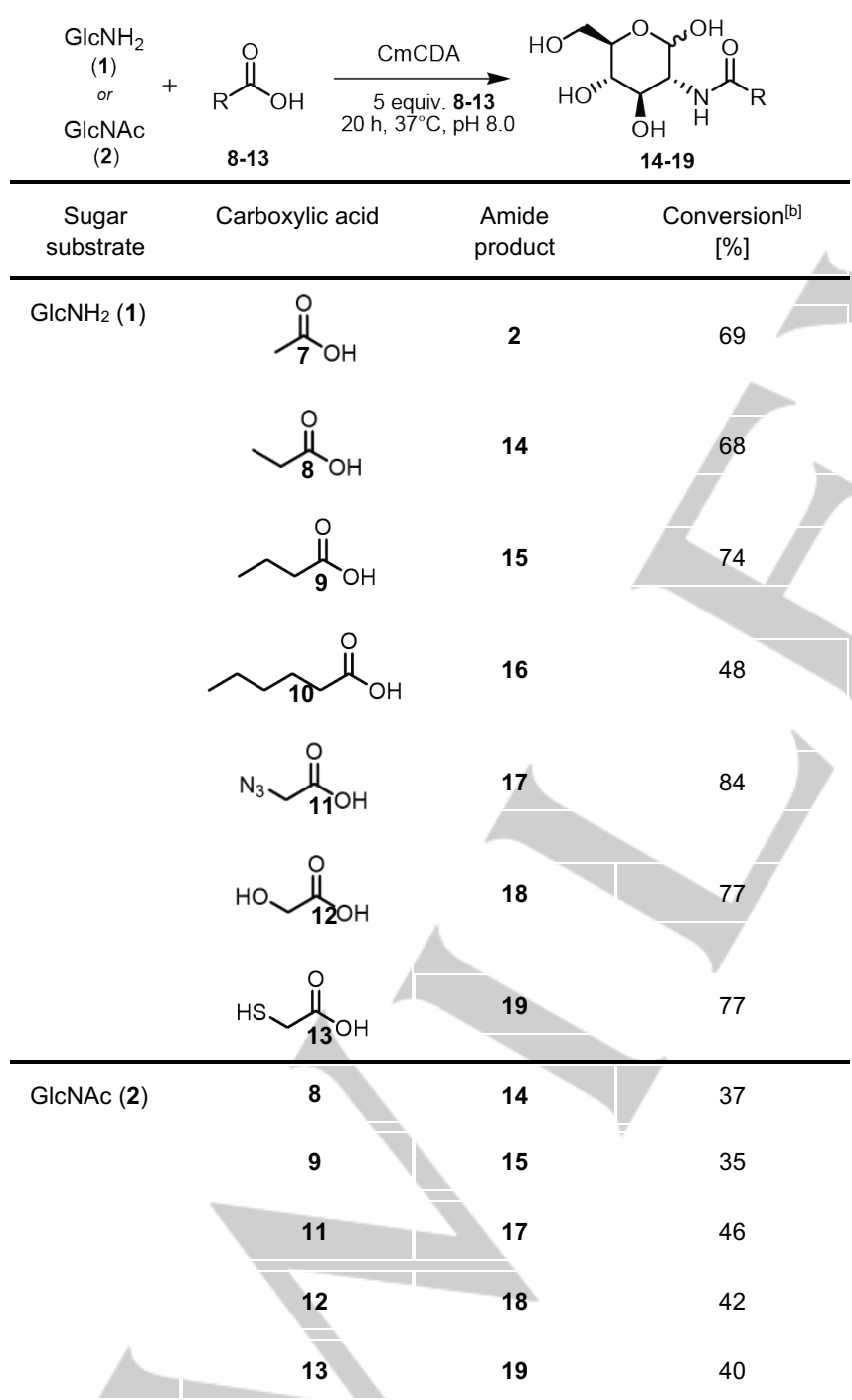

[a] $1 \mathrm{~mL}$ reactions consisting of $120 \mathrm{mM} 1$ or 2, $600 \mathrm{mM} \mathrm{7-13} \mathrm{(in} \mathrm{combination}$ with 1, or 8,9,11,12,13 with 2), $420 \mathrm{mM} \mathrm{Na}_{2} \mathrm{HPO}_{4}$ buffer ( $\mathrm{pH} \mathrm{8.0),} \mathrm{0.4} \mathrm{U} \mathrm{CmCDA,}$ $37^{\circ} \mathrm{C}, 20$ h. [b] Conversion determined by ${ }^{1} \mathrm{H}$ NMR.
To our delight CmCDA (expressed recombinantly in E.coli) could indeed catalyse $\mathrm{N}$-acylation reactions with a series of carboxylic acids: in the presence of 5 equivalents of acetic acid (7), propionic acid (8), butyric acid (9), hexanoic acid (10), azidoacetic acid (11), glycolic acid (12) and thioglycolic acid (13), reaction with glucosamine (2) yielded the corresponding $N$-acylglucosamines 2 and 14-19, respectively (Table 1 ).

Under the same conditions, no product formation was observed using benzoic acid as carboxylic acid donor, suggesting a steric or electronic limitation of CmCDA for bulkier aromatic organic acids. As expected, the amide formation requires an excess of the carboxylic acid in the reaction mixture: whereas 1 equivalent of acid gave low yield, the addition of 5 equivalents shifted the equilibrium to the formation of the amide bond.

To demonstrate the selectivity of CmCDA for glucosamine in the presence of other amines, 1 was reacted with an excess nonactivated, nonprotected $\mathrm{y}$-aminobutyric acid (20). Whereas no formation of $\mathrm{N}$ acyl glucosamine 21 could be observed using the inactive variant CmCDA-Asp21Asn [10], the formation of $\mathbf{2 1}$ catalysed by native CmCDA could be observed in a time-course experiment by LC-mass spectrometry (Scheme 3).
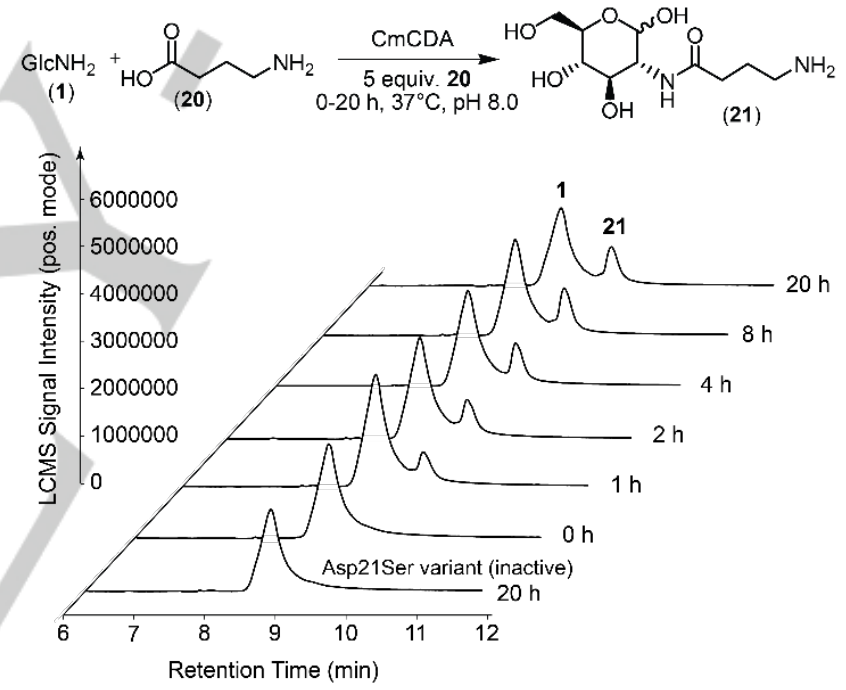

Scheme 3. LCMS analysis of CmCDA-catalysed $\mathrm{N}$-acylation reaction of 1 and amino acid 20 shows the formation of 21 [11].

Given that CmCDA also catalyses the hydrolysis, we then investigated whether it would be feasible to generate $N$-acyl glucosamine derivatives via $N$-transacylation directly from GlcNAc (1). Given the stability of the $N$-acyl bond, this had been achieved chemically before only by using highly fluorinated anhydrides: the exchange of the $\mathrm{N}$-acetyl group of unprotected and peracetylated GlcNAc to a $N$-perfluoroacyl group using trifluoroacetic anhydride or heptafluorobutanoic anhydride [12]. Further $N$-transacylation attempts used oleoyl chloride and lauryl chloride as acyl donors of protected GlcNAc in pyridine under reflux [13]. Examples of direct $N$ transacylation are limited, require harsh conditions and commonly the protection of all hydroxyl groups, and are restricted to fluorinated or long-chain acylation reagents. Performing these transacylation reactions with unprotected GIcNAc (1) and non-activated carboxylic acids enzymatically would extend the scope to acyl donors which are of interest in the synthesis of sialic acid derivatives, such as azido, hydroxyl or thiol groups [14]. 
Applying the reaction conditions established for the $\mathrm{N}$-acylation reaction of glucosamine (adding 5 equivalents of the acid donor), the overall efficiency of the $\mathrm{N}$-transacylation reaction was comparable to the conversion rates of the $\mathrm{N}$-acylation reactions (Table 1). Propionic acid (8), butyric acid (9), azidoacetic acid (11), glycolic acid (12) and thioglycolic acid (13) were explored as acyl donors and showed between $37-46 \%$ conversion to the corresponding $\mathrm{N}$-acyl glucosamines [15]. ${ }^{1} \mathrm{H}$ NMR analysis of the reaction mixtures showed the disappearance of $\mathbf{2}$ in all cases whereas $\mathbf{1}$ could be observed as product of the deacetylation reaction, suggesting that this enzymatic amine acyl exchange consists of two independent (faster) deacetylation and (slower) re-acylation steps.

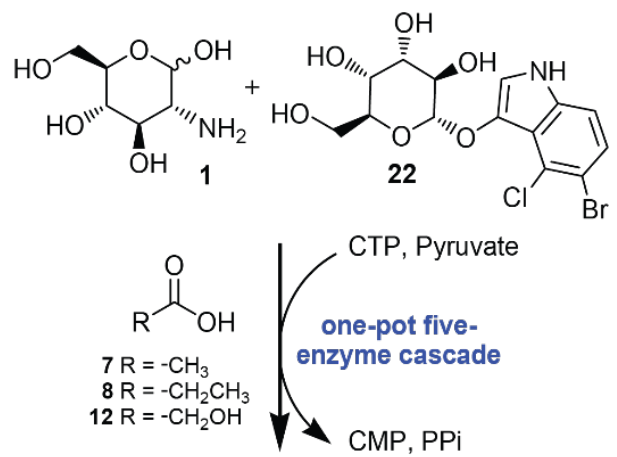

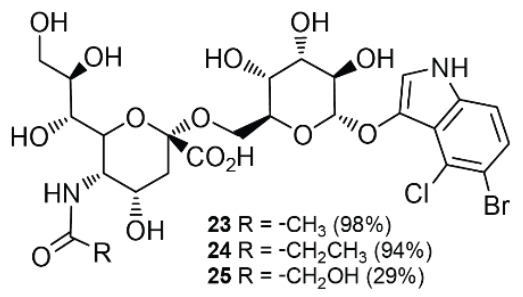

Scheme 4. One-pot five enzyme cascade for the synthesis of sialosides 23-25 from glucosamine and X-gal (22).

The enzymatic synthesis of C5-substituted sialosides requires $\mathrm{N}$ acylglucosamine or $\mathrm{N}$-acetylmannosamine derivatives as precursor molecules [16]. The enzymatic synthesis of these compounds would allow the integration of the $\mathrm{N}$-acylation reaction in established enzymatic synthesis cascades for unnatural sialosides, thus extending current one-pot enzyme cascades: in a first step, chemically $\mathrm{N}$-acylated glucosamine analogues are enzymatically epimerized to the corresponding $\mathrm{N}$-acylmannosamines and transformed into the sialic acid derivatives via an enzymatic aldol addition with pyruvate. These intermediates can be then used for the synthesis of sialyloligosaccharides via enzymatic activation by CMPsialic acid synthase and sialyltransferases. Previously, we had been successful in using 5-bromo-4-chloro-indolyl- $\beta$-D-galacto-pyranoside (X-Gal, 22) as an acceptor substrate for sialylation reactions, generating valuable labelled oligosaccharides. In the case of incomplete sialylation reactions, residual $\mathbf{2 2}$ could simply be removed by treating the reaction mixture with $\beta$-galactosidase. The released 5 bromo-4-chloro-3-indole dimerizes to form an insoluble indigo dye. Thus, sialosides containing $\mathrm{N}$-acetylneuraminic acid (Neu5Ac 23), $\mathrm{N}$ propanoylneuraminic acid (Neu5Prop 24) and $\mathrm{N}$-glycolylneuraminic acid (Neu5Gc 25) were synthesized using a five-enzyme one-pot reaction (Scheme 3 ) by only providing glucosamine (1), pyruvate, and acyl donors 7, 8 and 12 as sialic acid precursors, CTP for the activation of the sialic acid analogues, and $\mathbf{2 2}$ as the acceptor substrate for the sialylation reaction (Scheme 3). Sialosides 23 and 24 were obtained in over $94 \%$ yield, whereas 25 yielded $29 \%$ [17]. In conclusion, we have demonstrated that $\mathrm{CmCBA}$ is able to carry out a range of $\mathrm{N}$-acylation and $\mathrm{N}$-transacylation reactions to generate $\mathrm{N}$ acyl glucosamines on preparative scale. This enzyme is key for the development of comprehensive and extended enzymatic cascades, as demonstrated for the first enzymatic total synthesis of sialosides using glucosamine as sialic acid precursor, opening up new avenues in the chemoenzymatic synthesis of glycoconjugates and non-natural analogues.

\section{Acknowledgements}

This study was funded by the BBSRC, EPSRC, and InnovateUK: IBCatalyst programme BB/M029034/1, BB/M028836/1 (SLF), Marie Skłodowska-Curie Innovative Training Network H2020-MSCA-ITN2014-ETN-642870 (to SLF), the European Research Council (ERC) ProgrES-DVL-788231 (to SLF), the National Natural Science Foundation of China (NSFC) 31471703, 31671854, 31871793, and 31871754 (to JV and LL). We are grateful for the help provided by Prof. David Vocadlo (Burnaby, BC) for useful discussions on the catalytic mechanism of CmCDA.

Keywords: $\mathrm{N}$-acylation • enzymatic synthesis $\bullet$ unnatural sialosides - glycosylation $\cdot$ chitin de-N-acetylases

[1] a) K. M. Koeller, C. H. Wong, Chem. Rev. 2000, 100, 4465-4494. b) R. Sardzik, A. P. Green, N. Laurent, P. Both, C. Fontana, J. Voglmeir, M. J. Weissenborn, R. Haddoub, P. Grassi, S. M. Haslam, G. Widmalm, S. L. Flitsch, J. Am. Chem. Soc. 2012, 134, 4521-4524. c) J. Ye, H. Xia, N. Sun, C. C. Liu, A. Sheng, L. Chi, X. W. Liu, G. Gu, S. Q. Wang, J. Zhao, P. Wang, M. Xiao, F. Wang, H. Cao, Nat. Catal. 2019, 2, 514-522. d) K. Huang, S. L. Flitsch, Nat. Catal. 2019, 2, 479-480. e) H. Yu, X. Chen, Org. Biomol. Chem. 2016, 14, 2809-2818. f) K. W. Moremen, A. Ramiah, M. Stuart, J. Steel, L. Meng, F. Forouhar, H. A. Moniz, G. Gahlay, Z. Gao, D. Chapla, S. Wang, Jeong-Yeh Yang, Pradeep Kumar Prabhakar, Roy Johnson, M. Rosa, C. Geisler, A. V. Nairn, J. Seetharaman, S. C. Wu, L. Tong, H. J. Gilbert, J. LaBaer, D. L. Jarvis, Nat. Chem. Biol. 2018, 14, 156-162.

[2] a) C. Meng, A. Sasmal, Y. Zhang, T. Gao, C. C. Liu, N. Khan, A. Varki, F. Wang, H. Cao, Angew. Chem. 2018, 130, 9141-9145; Angew. Chem. Int. Ed. 2018, 57, 9003-9007. b) H. Yu, S. Huang, H. Chokhawala, M. Sun, H. Zheng, X. Chen, Angew. Chem. 2006, 118, 4042-4048; Angew. Chem. Int. Ed. 2006, 45, 3938-3944. c) S. Wang, Q. Zhang, C. Chen, Y. Guo, M. R. Gadi, J. Yu, U. Westerlind, Y. Liu, X. Cao, P. G. Wang, L. Li, Angew. Chem. 2018, 130, 9412-9417; Angew. Chem. Int. Ed. 2018, 57, 9268-9273. d) Z. P. Cai, L. P. Conway, Y. Y. Huang, W. J. Wang, P. Laborda, T. Wang, A. M. Lu, H. L. Yao, K. Huang, S. L. Flitsch, L. Liu, J. Voglmeir, Molecules 2019, 24, 1368. e) T. Heise, J. F. A. Pijnenborg, C. Bull, N. van Hilten, E. D. Kers-Rebel, N. Balneger, H. Elferink, G. J. Adema, T. J. Boltje, J. Med. Chem. 2019, 62, 1014-1021.

[3] a) D. J. Vocadlo, H. C. Hang, E. J. Kim, J. A. Hanover, C. R. Bertozzi, Proc. Natl. Acad. Sci. USA 2003, 100, 9116-9121. b) M. Boyce, I. S. Carrico, A. S. Ganguli, S. H. Yu, M. J. Hangauer, S. C. Hubbard, J. J. Kohler, C. R. Bertozzi, Proc. Natl. Acad. Sci. USA 2011, 108, 3141-3146. c) E. Memmel, A. Homann, T. A. Oelschlaeger, J. Seibel, Chem. Commun. 2013, 49, 7301-7303. d) Y. Zhu, J. Wu, X. Chen, Angew. Chem. 2016, 128, 9447-9451; Angew. Chem. Int. Ed. 2016, 55, 93019305. e) W. Qin, K. Qin, X. Fan, L. Peng, W. Hong, Y. Zhu, P. Lv, Y. Du, R. Huang, M. Han, B. Cheng, Y. Liu, W. Zhou, C. Wang, X. Chen, Angew. Chem. 2018, 130, 1835-1838; Angew. Chem. Int. Ed. 2018, 57, 18171820. f) A. P. Mattey, W. R. Birmingham, P. Both, N. Kress, K. Huang, J. M. van Munster, G. S. Bulmer, F. Parmeggiani, J. Voglmeir, J. E. R. Martinez, N. J. Turner, S. L. Flitsch, ACS Catal. 2019, 9, 8208-8212.[4] 
Y. M. Lv, P. Laborda, K. Huang, Z. P. Cai, M. Wang, A. M. Lu, C. Doherthy, L. Liu, S. Flitsch, J. Voglmeir, Green Chem. 2017, 19, 527-535.

[5] J. Choi, L. J. S. Wagner, S. B. P. E. Timmermans, S. A. Malaker, B. Schumann, M. A. Gray, M. F. Debets, M. Takashima, J. Gehring, C. R. Bertozzi, J. Am. Chem. Soc. 2019, 141, 13442-13453.

[6] a) Petchey, G. Grogan, Adv. Synth. Catal. 2019, doi 10.1002/adsc.201900694. b) A. J. L. Wood, N. J. Weise, J. D. Frampton, M. S. Dunstan, M. A. Hollas, S. R. Derrington, R. C. Lloyd, D. Quaglia, F. Parmeggiani, D. Leys, N. J. Turner, S. L. Flitsch, Angew. Chem. 2017, 129, 14690-14693; Angew. Chem. Int. Ed. 2017, 56, 14498-14501.

[7] a) C. P. Selitrennikoff, D. R. Sonneborn, Biochim. Biophys. Acta. 1976 451, 408-416. b) R. Hurtado-Guerrero, O. Raimi, S. Shepherd, D. M. van Aalten, FEBS Lett. 2007, 581, 5597-5600.

[8] a) R. Castangia, M. Austeri, S. L. Flitsch, Angew. Chem. 2012, 124 13191-13194; Angew. Chem. Int. Ed. 2012, 51, 13016-13018. b) N. P. Awasthi, R. P. Singh, J. Oleo. Sci. 2007, 56, 507-509.

[9] D. E. Blair, A. W. Schüttelkopf, J. I. MacRae, D. M. van Aalten, Proc. Natl. Acad. Sci. USA 2005, 102, 15429-15434.

[10] Details of the generation of the CmCDA Asp21Asn enzyme variant are described in the Supporting Information.

[11] $1 \mathrm{~mL}$ reaction were performed using $25 \mathrm{mM} \mathrm{GlcNH}_{2}$ (1), $125 \mathrm{mM}$ aminobutyric acid (20), $200 \mathrm{mM}$ Phosphate buffer, and 0.4 U CmCDA at $37^{\circ} \mathrm{C}$. Further details in the Supporting Information.

[12] a) B. Nilsson, S. Svensson, Carbohyd. Res. 1978, 62, 377-380. b) P. Rota, P. Allevi, R. Colombo, M. L. Costa, M. Anastasia, Angew. Chem. 2010, 122, 1894-1897; Angew. Chem. Int. Ed. 2010, 49, 1850-1853. c) G. Despras, A. Alix, D. Urban, B. Vauzeilles, J. M. Beau, Angew. Chem 2014, 126, 12106-12110; Angew. Chem. Int. Ed. 2014, 53, 11912-11916.
[13] a) Y. Yang, B. Yu, Tetrahedron Lett. 2007, 48, 7049-7052. b) I. García Álvarez, G. Corrales, E. Doncel Pérez, A. Muñoz, M. Nieto Sampedro, A. Fernández Mayoralas, J. Med. Chem. 2007, 50, 364-373.

[14] P. R. Wratil, R. Horstkorte, W. Reutter, Angew. Chem. 2016, 128, 96329665; Angew. Chem. Int. Ed. 2016, 55, 9482-9512.

[15] CmCDA catalysed conversion of GIcNAc (2) in the presence of propionic acid (8), butyric acid (9), azidoacetic acid (11), glycolic acid (12) and thioglycolic acid (13) into the corresponding $\mathrm{N}$-acylglucosamine derivatives 14,15 , and $17-19$ were monitored using ${ }^{1} \mathrm{H}$ NMR (Supporting Information).

[16] a) M. Knorst, W. D. Fessner, Adv. Synth. Catal. 2001, 343, 698-710. b) J. E. Reyes Martinez, R. Sardzik, J. Voglmeir, S. L. Flitsch, RSC Adv. 2013, 3, 21335-21338. c) H. Yu, K. Lau, V. Thon, C. A. Autran, E. Jantscher-Krenn, M. Xue, Y. Li, G. Sugiart, J. Qu, S. Mu, L. Ding, L. Bode, X. Chen, Angew. Chem. 2014, 126, 6805-6809; Angew. Chem. Int. Ed. 2014, 53, 6687-6691. d) A. Santra, A. Xiao, H. Yu, W. Li, Y. Li, L. Ngo, J. B. McArthur, X. Chen, Angew. Chem. 2018, 130, 2979-2983; Angew. Chem. Int. Ed. 2018, 57, 2929-2933.

[17] Enzymatic synthesis of $X$-gal sialoside using an one-pot five-enzyme cascade consisting of: a) CmCDA-catalysed $\mathrm{N}$-acylation to $\mathrm{N}$ acylglucosamines; b) PhGn2E-catalysed epimerisation to the corresponding mannosamine derivatives; c) EcAldolase -catalysed aldol reaction to $\mathrm{N}$-acylneuraminic acid derivatives; d) NmCTT-catalysed synthesis of cytidine-5-monophospho- $N$-acylneuraminic acid derivatives. e) PdST6-catalysed sialylation reaction of X-gal. Further details in the Supporting Information. 
WILEY-VCH

\section{Entry for the Table of Contents}

Layout 2:

\section{COMMUNICATION}<smiles>[R]C(=O)O</smiles>

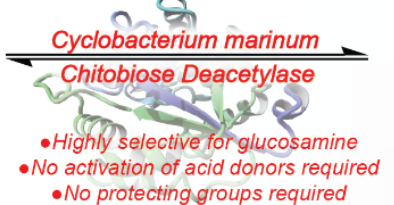

Selective N-acylation: The enzyme Chitobiose Deacetylase Cyclobacterium marinum was shown to have promiscuous $N$-acylation activity using a range of free carboxylic acids and glucosamine as substrates. This selective $\mathrm{N}$-acylation step provides the last missing link for the synthesis of natural and C5-modified sialosides from chitin and $\mathrm{N}$-acetyl glucosamine using a fully enzymatic reaction cascade.

Pedro Laborda, Yong-Mei Lyu, Fabio Parmeggiani, Ai-Min Lu, Ying-Ying Huang, Wen-Jiao Wang, Kun Huang, Juan Guo, Li Liu*, Sabine L. Flitsch*, and Josef Voglmeir*

Page No. - Page No.

Enzymatic N-Acylation Step Enables the Biocatalytic Synthesis of Unnatural Sialosides 